\title{
BMJ Open Quality Improving the prehospital management of ST elevation myocardial infarction: a national quality improvement initiative
}

\author{
Ian Howard, Nicholas Castle, Loua Al Shaikh, Robert Owen
}

To cite: Howard I, Castle N, Al Shaikh L, et al. Improving the prehospital management of ST elevation myocardial infarction: a national quality improvement initiative. BMJ Open Quality 2019;8:e000508. doi:10.1136/ bmjoq-2018-000508

Received 20 August 2018 Revised 20 November 2018 Accepted 20 November 2018

Check for updates

(C) Author(s) (or their employer(s)) 2019. Re-use permitted under CC BY-NC. No commercial re-use. See rights and permissions. Published by BMJ.

Critical Care Services, Hamad Medical Corporation Ambulance Service, Doha, Qatar

Correspondence to

Mr lan Howard, Hamad Medical Corporation Ambulance Service Doha Qatar ;

ianhoward@outlook.com

\section{ABSTRACT}

ST elevation myocardial infarction (STEMI) is a timedependent clinical emergency. Early recognition and intervention in the clinical course of STEMl are key to reducing mortality and morbidity. As a result, the benefits of the prehospital management of patients presenting with STEMI are well supported by the literature. Given these benefits, much of the focus on the development of quality and performance measures for Emergency Medical Services has focused on STEMI care. Historically, within Qatar, however, no measures of prehospital STEMI care have previously existed and as such, little is understood regarding the quality of prehospital care delivered to patients with STEMI. The overall aim of this national initiative was to improve the effectiveness of the prehospital care of patients with STEMI, to a minimum compliance of $75 \%$, as measured by four process measures and one bundle measure, over a 12-month period. Initial efforts were aimed at the development of relevant indicators to guide assessment and identifying an appropriate patient cohort to test improvement efforts. Using these measures and criteria, the project team highlighted several areas for potential improvement centred on three key domains within the service: clinical practice, training and clinical equipment/medication. There was significant and sustained improvement across all measures recorded. For the bundle measure, the median proportional compliance increased from $39 \%$ pre-improvement activities to $76 \%$ post-improvement activities and remained sustained at 12 months postimplementation. The initiative was successful in meeting all of its aims and furthermore showed sustained compliance at 12 months post-implementation, thanks in part to what were designed to be changes that were simple, yet pragmatic, and readily producible at scale. While a formal cost analysis was not conducted, the improvement activities capitalised on existing organisational structures and processes with the resultant cost perceived to be negligible.

\section{PROBLEM}

ST elevation myocardial infarction (STEMI) is a time-dependent clinical emergency. Early recognition and intervention in the clinical course of STEMI are key to reducing mortality and morbidity. As a result, the benefits of the prehospital management of patients presenting with STEMI are well supported by the literature. Given these benefits, much of the focus on the development of quality and performance measures for Emergency Medical Services (EMS) has focused on STEMI care. Historically, within Qatar, however, no measures of prehospital STEMI care have previously existed and as such, little is understood regarding the quality of prehospital care delivered to patients with STEMI.

The overall aim of this national initiative was to improve the effectiveness of the prehospital care of patients with STEMI, to a minimum compliance of $75 \%$, as measured by four process measures and one bundle measure, over a 12-month period.

\section{Setting}

Hamad Medical Corporation Ambulance Service (HMCAS) is the national ambulance service of the State of Qatar and responds to approximately 800-1000 combined emergency and non-emergency/transfer cases a day. The ambulance operates a two-tiered system of Ambulance Paramedic staffed ambulances and Critical Care Paramedic staffed fast-response vehicles.

The majority of the population of Qatar is composed of expatriate workers, with Qatari citizens making up approximately 15\%-20\% of the population. The diverse mix of nationalities and cultures among the populace is echoed in the broader healthcare workforce. This has added an extra layer of complexity in day-to-day operations of delivering healthcare in the country, given the diverse backgrounds in education and experience over and above the ethnic, cultural and language differences among healthcare workers in Qatar.

Within the ambulance service, Critical Care Paramedics are recruited from EMS organisations around the world with specific training and experience in prehospital emergency care. Ambulance Paramedics, however, are sourced from a variety of allied healthcare backgrounds and then receive additional in-service bridging training once in country. Given this, a large majority of EMS staff would 
not have had prehospital emergency care experience prior to their employment within the HMCAS.

\section{BACKGROUND}

The incidence and burden of ischaemic heart disease on disability-adjusted life years and life expectancy, worldwide, is significant. ${ }^{12}$ Early recognition and intervention are pivotal in the acute and emergent presentation of ischaemic heart disease and has the potential to improve patient experience, prevent deaths and reduce long-term morbidity. ${ }^{3}$ As a result, the role of EMS for the initiation of basic care for these patients is well supported by the literature. ${ }^{45}$ These services are poised to play a progressively crucial role in this context, as they increasingly act as the entry point into the broader healthcare system for these patients into the future.

There is limited evidence to suggest variation and several shortfalls in the prehospital management of patients with STEMI. ${ }^{67}$ The apparent causes of this are broad and have been attributed to a combination of patient, provider and institutional factors. ${ }^{8}$ Despite this, targeted improvement initiatives have shown success in improving the overall quality in the prehospital management of patients presenting with STEMI. ${ }^{7}$

Within Qatar, this incidence and impact of ischaemic heart disease and its acute manifestations is no different. As a group of patients identified as high risk, and for which a larger integrated pathway has recently been developed within the broader government health system, HMCAS wanted to identify the quality of care delivered to these patients and further explore improving care if found to be deficient.

\section{MEASUREMENT}

Prehospital care delivered by EMS is largely symptomatic in nature and so lends itself to assessment by quality indicators. This is evident in the high proportion of processbased measures of care that dominate the literature surrounding prehospital quality assessment. ${ }^{9}$ Outcomes, however, are more difficult to assess due to the limited time in which patients are exposed to EMS care. As a proxy, composite bundle measures are often assembled from several process measures in order to assess EMS-specific patient outcomes. ${ }^{69}$

Prior to the start of the project, no baseline measurement had been carried out, as no STEMI-specific measures of quality were in use in HMCAS. Initial efforts were therefore aimed at the development of relevant indicators to guide assessment and identification of an appropriate patient cohort to monitor improvement efforts.

A basic literature review was conducted in order to identify measures of care for potential use. Given the strength of evidence supporting the prehospital care of patients with STEMI, a significant body of literature dedicated to the development of STEMI-based quality indicators already existed. ${ }^{9}$ Several indicators were developed

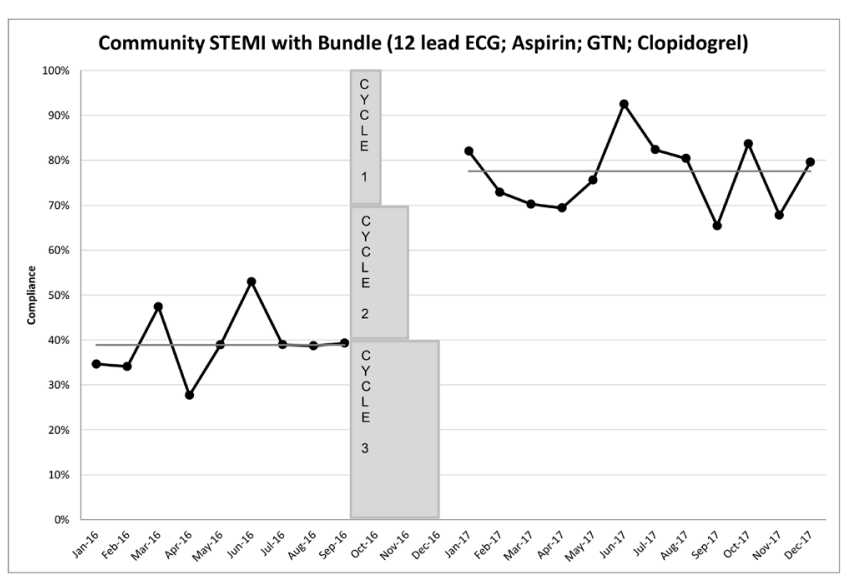

Figure 1 Bundle compliance. GTN, glyceryl trinitrate; STEMI, ST elevation myocardial infarction.

from the literature, adapted for local use and included four process measures and one bundle measure:

- Patients with a provisional diagnosis of STEMI who received a 12 lead ECG (QI1). ${ }^{1}$

- Patients with a provisional diagnosis of STEMI who were administered aspirin (QI2). ${ }^{2}$

- Patients with a provisional diagnosis of STEMI who were administered glyceryl trinitrate (GTN) (QI3). ${ }^{3}$

- Patients with a provisional diagnosis of STEMI who were administered clopidogrel (QI4). ${ }^{4}$

- Composite bundle measure consisting of all four process measures (QI1+QI2+QI3+QI4).

Running concurrently, the HMCAS patient care database was scrutinised to identify appropriate inclusion and exclusion criteria against which the indicators could be applied. A final set of criteria was developed over several months and accounts for the initial variation in patients included for assessment over the first few months of the project (see figure 1). The final list included:

- Inclusion criteria:

Patients with a provisional diagnosis of STEMI (including all variations, eg, anterior, inferior, etc)

- Exclusion criteria:

Patients with an initial systolic blood pressure $<90 \mathrm{~mm}$ $\mathrm{Hg}$.

Patients originating from a healthcare facility, that is, non-community cases.

Historically, patient care data were captured by practitioners on paper-based patient care records. Key data fields were later manually entered into an electronic database by dedicated clinical document officers. Approximately halfway through the improvement project, an electronic patient care record was implemented into the service. This was used as a natural break in the project to allow for the introduction of improvement activities.

\section{DESIGN}

A clinical governance project team was assembled consisting of three senior clinical managers, with ad hoc 
inclusion as required by the Medical Director and/or other Executive representation; a representative from Operations Management and a representative from Medical Stores/Supplies. In addition, throughout the development phase, an Improvement Advisor from the Institute for Healthcare Improvement was included in the project team to provide ongoing advice and consultation regarding indicator development and measurement and the design and implementation of improvement activities. Initial efforts were aimed at the development of relevant indicators to guide assessment and identifying an appropriate patient cohort to test improvement efforts. Using these measures and criteria, the project team highlighted several areas for potential improvement centred on three key domains within the service: clinical practice, training and clinical equipment/medication.

During the initial months, the team met monthly to gauge progress and plan the activities for the coming weeks. Once the improvement activities had started, the meetings moved to a quarterly basis with ad hoc meetings as and when required. For reporting purposes, proportion compliance for all measures was calculated monthly and plotted on run charts with median proportion compliance additionally plotted.

\section{STRATEGY}

The overall aim was to improve the efficiency of the prehospital care of patients with STEMI to a minimum compliance of $75 \%$, as measured by four process measures and one bundle measure, over a 12-month period. To achieve this, improvement activities were divided into three phases, with each one representing an implementation cycle. Several activities ran concurrently, based on their anticipated time frame for execution, as well as to maximise the impact of the individual activities.

- Implementation cycle 1/practice review: clinical practice guidelines were updated to reflect contemporary changes in the prehospital management of STEMI. ${ }^{10} 11$ The addition of GTN and clopidogrel were added to the Ambulance Paramedics scope of practice, and a decision tree to guide the use of 12 lead ECG monitoring and diagnosis was additionally included. Previous HMCAS guidelines had been conservative in the use of GTN in particular; however, following a brief evidence review, its use was encouraged and the blood pressure limits cautioning its use relaxed as a result of this review ${ }^{1011}$ (figure 2).

- Implementation cycle 2/equipment review: a drug prepack was assembled consisting of oral aspirin tablets, oral clopidogrel tablets and a bottle of GTN

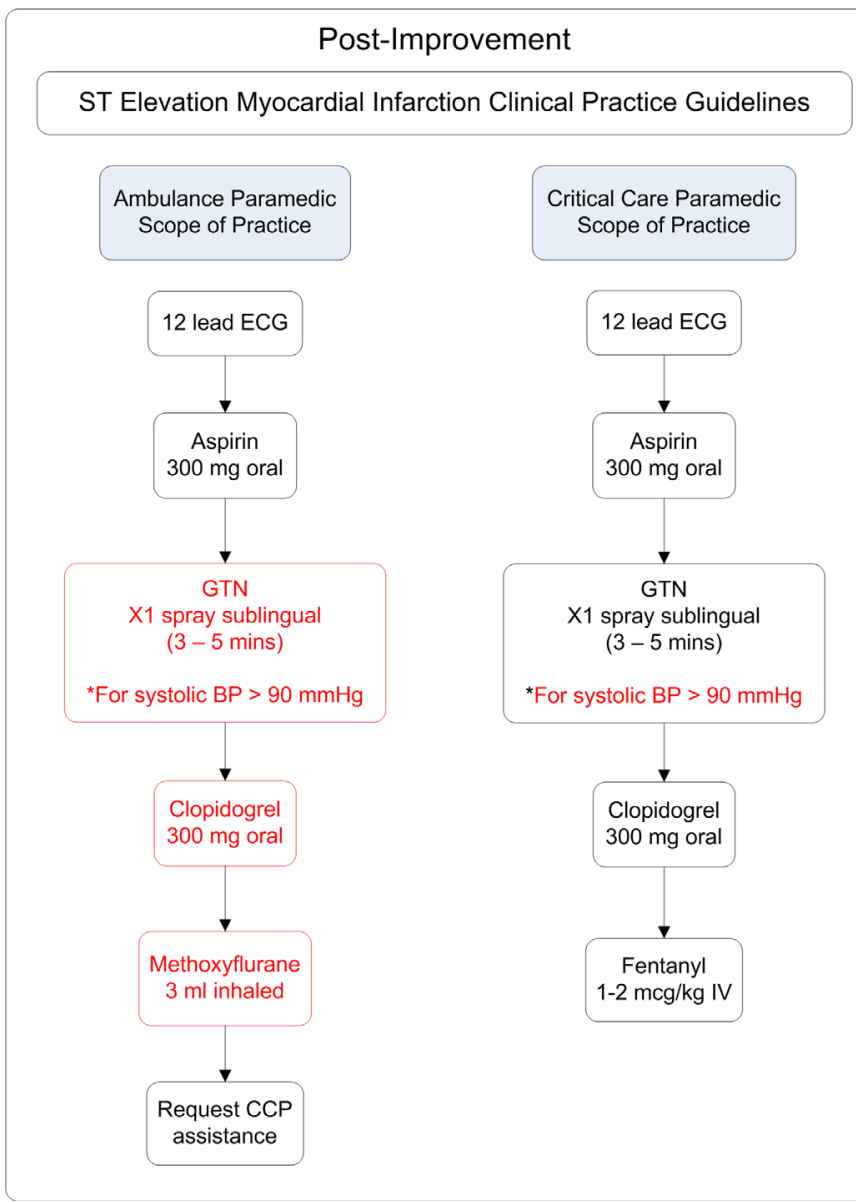


Table 1 Results summary

\begin{tabular}{|c|c|c|c|c|}
\hline & $\begin{array}{l}\text { Baseline/pre- } \\
\text { implementation }\end{array}$ & $\begin{array}{l}\text { Baseline median } \\
\text { compliance }\end{array}$ & Post-implementation & $\begin{array}{l}\text { Post-implementation } \\
\text { median compliance }\end{array}$ \\
\hline Total transports & 473 & & 601 & \\
\hline Aspirin & 351 & $74 \%$ & 518 & $86 \%$ \\
\hline Glyceryl trinitrate & 250 & $53 \%$ & 475 & $79 \%$ \\
\hline
\end{tabular}

spray to serve as an aide-memoire for the pharmacological management of patients with STEMI. This was packed together with a dosing card in all operational Ambulance Paramedic and Critical Care Paramedic drug bags for distribution by a central pharmacy store.

- Implementation cycle 3/training review: lastly, mandatory in-service continued professional development training had been introduced prior to the start of the improvement project. This was capitalised on to both introduce the new guidelines for the management of STEMI and serve as a general clinical refresher for STEMI care and ECG diagnosis.

\section{RESULTS}

There was significant and sustained improvement across all measures recorded. The table 1 highlights the median proportional changes in STEMI care compliance pre-improvement and post-improvement activities, for all measures developed for the project period. For the bundle measure, the median proportional compliance increased from $39 \%$ pre-improvement activities to $76 \%$ post-improvement activities and remained sustained at 12 months post-implementation (figure 1).

Of interest to note, being a composite measure, the performance of the bundle indicator post-improvement was entirely dependent on the weakest single indicator that contributed towards the bundle, in this case, the administration of clopidogrel. Furthermore, beyond coincidence, the administration of clopidogrel acted as a 'predictor' of bundle success - that is, all patients who received clopidogrel received all the other components of the bundle. This, however, was not entirely surprising, given that the addition of clopidogrel to the Ambulance Paramedic scope of practice constituted the biggest pragmatic change in the improvement initiative.

The improvement in compliance has been attributed largely to the changes in the Ambulance Paramedic scope of practice, supplemented with the focus of these changes into continued professional development activities, and assisted to a lesser extent by the new drug prepack. The focus for dispatch to STEMI cases within HMCAS had been to deliver a Critical Care Paramedic to all cases of STEMI, regardless of acuity. However, this was not always possible/and or varied. As a result, historically, patients who did not receive care by a Critical Care Paramedic would not have received the requisite minimum standard expected for prehospital STEMI care. With the changes to the scope of practice, whether a Critical Care Paramedic was available or not, the required minimum care was available to Ambulance Paramedics to deliver to patients with STEMI.

As a surrogate balancing measures of sorts, a sample of the post-implementation cases transported by Ambulance Paramedics alone were manually reviewed for potential adverse events, given the changes to their scope of practice. Zero clinical adverse events were found that could have potentially occurred because of these practice changes. Similarly, EMS call to Emergency Department times were measured and compared in a sample of cases both pre-improvement and post-improvement activities, with little to no difference found between each group.

\section{Lessons and limitations}

Qatar is a relatively small country, with an equally small population. Despite a proportionally high healthcare service use, organisational barriers were few, and introducing broad service wide changes in this setting were perceived to be simpler and achieved relatively quicker as could be expected with a bigger service and/or setting. This was felt to additionally contribute to the impact each activity had towards achieving the overall initiative goal.

The implementation phase of the organisation's electronic patient care record coincided with the planned introduction of the improvement activities. For the initial 3 months (Oct 2016-Dec 2016), there were significant difficulties in accessing the required data and concerns around data quality due to the transition from paper to electronic records. As a result, data collection was abandoned for this period. This, however, proved fortuitous in that it served as a natural break in the data measurement to not only introduce and maximise the improvement activities but also reinforce the impact of these changes when displayed graphically.

Lastly, despite improvements in all measures, the increase was unequal across the measures. It was possible that several 'administrative' factors such as poor documentation and/or inaccurate inclusion and exclusion criteria could have exerted an influence; however, this also represents the best avenue for further research in order to understand reasons behind the unequal compliance. 


\section{CONCLUSION}

STEMI is a time-dependent clinical emergency, for which early recognition and intervention are crucial towards reducing mortality and morbidity. Consequently, the role of EMS in the initiation of basic care for these patients is well supported by the literature. There is limited evidence, however, that has previously observed variation in the prehospital care of patients with STEMI. Despite this, with targeted improvement activities, underpinned by focused quality assessment, the delivery of high-quality care is achievable.

The aim of this improvement initiative was to increase the effectiveness of prehospital care of patients with STEMI, as measured by several key indicators of care. However, no previous baseline measurement existed within HMCAS, the participating service. As a result, the development and implementation of a suite of measures was included as part of the project. Three key areas were identified and targeted for improvement activities and included: clinical practice; training and clinical equipment/medication.

The initiative was successful in meeting all of its aims and furthermore showed sustained compliance at 12 months post-implementation, thanks in part to what were designed to be changes that were simple, yet pragmatic, and readily producible at scale. While a formal cost analysis was not conducted, the improvement activities capitalised on existing organisational structures and processes with the resultant cost perceived to be negligible.

Acknowledgements The authors wish to thank David Williams for his invaluable input during the early phases of the project, for guidance in identifying relevant and appropriate quality indicators and for input regarding the improvement activities.

Contributors All authors conceived the idea for the research and contributed towards data collection, analysis and the final draft manuscript for submission. IH was primarily responsible for the draft of the manuscript.

Funding The authors have not declared a specific grant for this research from any funding agency in the public, commercial or not-for-profit sectors.

Competing interests None declared.

Patient consent for publication Not required.
Provenance and peer review Not commissioned; externally peer reviewed.

Open access This is an open access article distributed in accordance with the Creative Commons Attribution Non Commercial (CC BY-NC 4.0) license, which permits others to distribute, remix, adapt, build upon this work non-commercially, and license their derivative works on different terms, provided the original work is properly cited, appropriate credit is given, any changes made indicated, and the use is non-commercial. See: http://creativecommons.org/licenses/by-nc/4.0/.

\section{REFERENCES}

1. Vos T, Abajobir AA, Abate $\mathrm{KH}$, et al. Global, regional, and national incidence, prevalence, and years lived with disability for 328 diseases and injuries for 195 countries, 1990-2016: a systematic analysis for the Global Burden of Disease Study 2016. The Lancet 2017;390:1211-59.

2. GBD 2016 Causes of Death Collaborators. Global, regional, and national age-sex specific mortality for 264 causes of death, 19802016: a systematic analysis for the Global Burden of Disease Study 2016. Lancet 2017;390:1151-210.

3. Bång A, Grip L, Herlitz J, et al. Lower mortality after prehospital recognition and treatment followed by fast tracking to coronary care compared with admittance via emergency department in patients with ST-elevation myocardial infarction. Int J Cardiol 2008;129:325-32.

4. O'Gara PT, Kushner FG, Ascheim DD, et al. 2013 ACCF/AHA guideline for the management of ST-Elevation myocardial infarction. J Am Coll Cardiol 2013;61:e78-e140.

5. Ibanez B, James S, Agewall S, et al. 2017 ESC Guidelines for the management of acute myocardial infarction in patients presenting with ST-segment elevation The Task Force for the management of acute myocardial infarction. Eur Heart J 2018;39:119-77.

6. Siriwardena AN, Shaw D, Donohoe R, et al. Development and pilot of clinical performance indicators for English ambulance services. Emerg Med J 2010;27:327-31.

7. Siriwardena AN, Shaw D, Essam N, et al. The effect of a national quality improvement collaborative on prehospital care for acute myocardial infarction and stroke in England. Implement Sci 2014;9:1-9

8. Togher FJ, Davy Z, Siriwardena AN. Patients' and ambulance service clinicians' experiences of prehospital care for acute myocardial infarction and stroke: a qualitative study. Emerg Med $J$ 2013;30:942-8.

9. Howard I, Cameron P, Wallis L, et al; Quality indicators for evaluating prehospital emergency care: a scoping review, 2017.

10. Ibanez B, James S, Agewall S. 2017 ESC Guidelines for the management of acute myocardial infarction in patients presenting with ST -segment elevation The Task Force for the management of acute myocardial infarction. Eur Heart J 2018;39:119-77.

11. O'Gara PT, Kushner FG, Ascheim DD, et al. 2013 ACCF/AHA guideline for the management of ST-elevation myocardial infarction: a report of the American College of Cardiology Foundation/American Heart Association Task Force on Practice Guidelines. Circulation 2013;127:e362-425. 